\title{
ON A NONLINEAR EQUATION OF DAMPED OSCILLATIONS BEAM
}

\author{
Salavat Gilmanov ${ }^{1}$, Andrey Akimov ${ }^{2}$, Guzeliya Galiaskarova ${ }^{3}$ \\ ${ }^{1,2,3}$ Sterlitamak Branch \\ Bashkir State University \\ Sterlitamak, RUSSIA
}

\begin{abstract}
The article considers the problem of damping vibrations of the beam for a nonlinear equation in the case of rigidly fixed ends. It is proved that solution to a nonlinear beam equation is uniquely. It is shown that under certain conditions on the coefficients of the solution of the problem will be oscillating.
\end{abstract}

AMS Subject Classification: $35 \mathrm{M} 12$

Key Words: damped oscillations of a beam, oscillatory solutions, initial and boundary conditions

\section{Introduction}

Many problems on vibrations of rods, beams and plates, which are of great importance in structural mechanics lead to differential equations of higher order than the string equation. The first study of beams on the Winkler foundation subjected to a simple concentrated force moving with a constant speed was initiated by Timoshenko [2]. We consider the extensible beam equation and investigate oscillations of extensible beam equations

$$
\frac{\partial^{2} u}{\partial t^{2}}+\alpha \frac{\partial^{4} u}{\partial x^{4}}-\left(\beta+\gamma \int_{0}^{L}\left(\frac{\partial u(\xi, t)}{\partial \xi}\right)^{2} d \xi\right)^{2} \frac{\partial^{2} u}{\partial x^{2}}+c(x, u)=f
$$

in a cylindrical domain $\Omega=I \times(0, \infty)$, where $f=f(x, t)$ and $\alpha, \beta, \gamma, L$ are

Received: November 30, 2015

Published: February 18, 2016

${ }^{\S}$ Correspondence author (c) 2016 Academic Publications, Ltd.

url: www.acadpubl.eu 
constants such that $\alpha>0, \gamma \geq 0, L>0$ and $I=(0, L)$. We deal with the case of clamped ends for which

$$
u(0, t)=\frac{\partial u}{\partial x}(0, t)=u(L, t)=\frac{\partial u}{\partial x}(L, t)=0, t>0,
$$

and initial data

$$
u(x, 0)=\varphi(x), \quad u_{t}(x, 0)=\psi(x), \quad x \in(0, L) .
$$

\section{Theorem of uniqueness}

Theorem 1. Assume that solution of problem (1)-(3) exists and i) $\alpha>$ $0, \beta>0, \gamma>0 ; i i) \int_{0}^{p} c(x, s) d s \geq 0$ then solution is uniquely in the class $u(x, t) \in C(\bar{\Omega}) \bigcap C_{x, t}^{4,2}(\bar{\Omega})$.

Proof. Suppose that there are two solutions $u_{1}$ and $u_{2}$ of the problem (1)(3). We introduce the function $u=u_{1}-u_{2}$. The function $u$ satisfies the equation (1), the boundary conditions (2) and the homogeneous initial data (3). Consider the "energy integral", which is the sum of the kinetic energy, potential energy of the transverse oscillations, the potential energy of the torsion and energy of power of resistance

$$
\begin{aligned}
F(t) & =\frac{1}{2}\left[\int_{0}^{L} u_{t}^{2} d x+\alpha \int_{0}^{L} u_{x x}^{2} d x+\beta \int_{0}^{L} u_{x}^{2} d x\right]^{+} \\
+ & {\left[\frac{\gamma}{2}\left(\int_{0}^{L} u_{x}^{2} d x\right)+\int_{0}^{L} \int_{0}^{u(x, t)} c(x, s) d s d x\right] . }
\end{aligned}
$$

Differentiating the function $F(t)$ to obtain

$$
\begin{aligned}
F^{\prime}(t) & =\int_{0}^{L} u_{t} u_{t t} d x+\alpha \int_{0}^{L} u_{x x} u_{x x t} d x+\beta \int_{0}^{L} u_{x} u_{x t} d x+ \\
& +\gamma \int_{0}^{L} u_{x}^{2} d x \int_{0}^{L} u_{x} u_{x t} d x+\int_{0}^{L} c(x, u(x, t)) u_{t} d x .
\end{aligned}
$$


Integrating this expression by parts with boundary conditions (2) to obtain

$$
\begin{aligned}
& \int_{0}^{L} u_{x x} u_{x x t} d x=\int_{0}^{L} u_{x x x x} u_{t} d x, \\
& \int_{0}^{L} u_{x} u_{x t} d x=-\int_{0}^{L} u_{x x} u_{t} d x .
\end{aligned}
$$

Combining (4)-(6) with (1), we obtain

$$
F^{\prime}(t)=\int_{0}^{L} u_{t}\left(u_{t t}+\alpha u_{x x x x}-\left(\beta+\gamma \int_{0}^{L} u_{x}^{2} d x\right) u_{x x}+c(x, u)\right) d x=0
$$

Consequently we have $F(t)=$ const. Since $F(0)=0$ then $F(t)=0$ and $u(x, t) \equiv 0$.

\section{Theorem about oscillatory of solution}

Definition 1. The function $u(x, t)$ is said eventually globally oscillatory if for any $T \geq 0$ there exist $\left(x_{1}, t_{1}\right) \in \times I(T ;+\infty)$ such that $u\left(x_{1}, t_{1}\right)>0$ and simultaneously there exist $\left(x_{2}, t_{2}\right) \in I \times(T ;+\infty)$ such that $u\left(x_{2}, t_{2}\right)<0$ or $u(x, t) \equiv 0$ for some interval $(T ;+\infty)$.

The following theorem is almost identical to theorem from [3], [4]

Theorem 2. Assume that: (i) $c(x, \xi) \in C(I \times R)$ and $\xi c(x, \xi) \geq$ 0 for $(x, \xi) \in I \times R, \xi \in R$; (ii) $f(x, t) \in C(\bar{\Omega})$; (iii) there exists a function $\theta(x) \in$ $C^{4}(I)$ such that $\alpha \theta^{(4)}(x)-\beta \theta^{\prime \prime}(x) \geq k \theta(x)$, in $I$ for some constant $k \geq 0$ $\theta^{\prime \prime}(x) \leq 0$ in $I, \theta(0)=\theta(L)=\theta^{\prime}(0)=\theta^{\prime}(L)=0$.

Every solution $u \in C^{4}(\bar{\Omega} ; R)$ of the boundary value problem (1), (2) is oscillatory in $\Omega$ if the ordinary differential inequalities

$$
y^{\prime \prime}+k y \leq \pm \int_{0}^{L} f(x, t) \theta(x) d x
$$

have no eventually positive solutions. 
Proof. Suppose to the contrary that there is a solution $u \in C^{4}(\bar{\Omega})$ of the problem (1), (2) which has no zero in $I \times\left(t_{0}, \infty\right)$ for some $t_{0}>0$. First we assume that $u>0$ in $I \times\left(t_{0}, \infty\right)$. Since $c(x, u) \geq 0$ in $I \times\left(t_{0}, \infty\right)$, we have for $(x, t) \in I \times\left(t_{0}, \infty\right)$

$$
\frac{\partial^{2} u}{\partial t^{2}}+\alpha \frac{\partial^{4} u}{\partial x^{4}}-\left(\beta+\gamma \int_{0}^{L}\left(\frac{\partial u(\xi, t)}{\partial \xi}\right)^{2} d \xi\right) \frac{\partial^{2} u}{\partial x^{2}} \leq f(x, t)
$$

We multiply (8) by $\theta(x)$ and then integrate over $I$ to obtain

$$
\begin{gathered}
\int_{0}^{L} \frac{\partial^{2} u}{\partial t^{2}} \theta(x) d x+\alpha \int_{0}^{L} \frac{\partial^{4} u}{\partial x^{4}} \theta(x) d x-\int_{0}^{L} \beta \frac{\partial^{2} u}{\partial x^{2}} \theta(x) d x- \\
-\gamma \int_{0}^{L}\left(\frac{\partial u(\xi, t)}{\partial \xi}\right)^{2} d \xi \int_{0}^{L} \frac{\partial^{2} u}{\partial x^{2}} \theta(x) d x \leq \int_{0}^{L} f(x, t) \theta(x) d x .
\end{gathered}
$$

Integration by parts yields

$$
\begin{aligned}
& \int_{0}^{L} \frac{\partial^{2} u}{\partial x^{2}} \theta(x) d x=\int_{0}^{L} u \theta^{\prime \prime}(x) d x, \quad t>t_{0}, \\
& \int_{0}^{L} \frac{\partial^{4} u}{\partial x^{4}} \theta(x) d x=\int_{0}^{L} u \theta^{(4)}(x) d x, \quad t>t_{0},
\end{aligned}
$$

Combining (9)-(11), we obtain

$$
\begin{gathered}
\frac{d^{2}}{d t^{2}} \int_{0}^{L} u \theta(x) d x+\int_{0}^{L} u\left(\alpha \theta^{(4)}(x)-\beta \theta^{\prime \prime}(x)\right) d x- \\
-\gamma \int_{0}^{L}\left(\frac{\partial u(\xi, t)}{\partial \xi}\right)^{2} d \xi \int_{0}^{L} u \theta^{\prime \prime}(x) d x \leq \int_{0}^{L} f(x, t) \theta(x) d x, t>t_{0}
\end{gathered}
$$

and therefore

$$
U^{\prime \prime}(t)+k U(t) \leq \int_{0}^{L} f(x, t) \theta(x) d x, t>t_{0}
$$




$$
U(t)=\int_{0}^{L} u \theta(x) d x
$$

Consequently, we observe that $U(t)$ is a positive solution of

$$
y^{\prime \prime}+k y \leq \pm \int_{0}^{L} f(x, t) \theta(x) d x, \quad t>t_{0}
$$

This contradicts the hypothesis. If $u<0$ in $I \times\left(t_{0}, \infty\right), v:=-u$ satisfies

$$
\frac{\partial^{2} v}{\partial t^{2}}+\alpha \frac{\partial^{4} v}{\partial x^{4}}-\left(\beta+\gamma \int_{0}^{L}\left(\partial \frac{\partial v(\xi, t)}{\partial \xi}\right)^{2} d \xi\right) \frac{\partial^{2} v}{\partial x^{2}} \leq-f(x, t)
$$

Proceeding as in the case where $u>0$, we are led to a contradiction. The proof is complete.

If $\alpha \mu^{4}+\beta \mu^{2} \geq 0$, where $\mu$ the smallest positive root of the equation $\cos (\mu L) \operatorname{ch}(\mu L)=1$, it is easy to see $\theta(x)=(\operatorname{sh}(\mu L)-\sin (\mu L))(\cos (\mu x)-$ $\operatorname{ch}(\mu x))-(\operatorname{ch}(\mu L)-\cos (\mu L))(\sin (\mu x)-\operatorname{sh}(\mu x))$ satisfies the hypothesis (iii) of Theorem 1 with $k=\alpha \mu^{4}+\beta \mu^{2}$. The following statement is almost identical to statement from [3], [4]

Corollary 1. Assume that the hypotheses (i) and (ii) of Theorem 2 are satisfied, and that $\alpha \mu^{4}+\beta \mu^{2} \geq 0$. Every solution $u \in C^{4}(\bar{\Omega})$ of the problem (1), (2) is oscillatory in $\Omega$ if

$$
\begin{gathered}
\lim _{t \rightarrow \infty} \inf \int_{T}^{t}\left(1-\frac{s}{t}\right) F(s) d s=-\infty \\
\lim _{t \rightarrow \infty} \sup _{T} \int_{T}^{t}\left(1-\frac{s}{t}\right) F(s) d s=\infty
\end{gathered}
$$

for all large $T$, where

$$
F(t)=\int_{0}^{L} f(x, t) \theta(x) x d x
$$




\section{References}

[1] Feireisl E. and Herrmann L. Oscillations of a nonlinearly damped extensible beam, Appl. Math., 37, (1991), pp. 469-478.

[2] S. Timoshenko D. H. Young and W. Weaver Jr. Vibration Problems in Engineering, John Wiley Sons, New York, NY, USA, 4th edition, (1974).

[3] Yoshida N. Forced oscillations of nonlinear extensible beams, Proceedings of the Tenth International Conference on Nonlinear Oscillations, Varna, (1984), pp. 814-817. doi:10.1137/0516016.

[4] Yoshida N. On the zeros of solutions of beam equations, Ann. Mat. Pura Appl., 151, (1988), pp. 389-398. doi 10.1007/BF01762806.

[5] Akimov A. A. On uniqueness Morawetz problem for the Chaplygin equation, International Journal of Pure and Applied Mathematics, 97, No. 3, (2014), 369-375. doi:10.12732/ijpam.v97i3.9.

[6] Akimov A. Galiaskarova, G. The solution of the Darboux problem for the telegraph equation with deviation from the characteristic, International Journal of Pure and Applied Mathematics, 103, No. 2, (2015), 377-383. doi:10.12732/ijpam.v103i2.20.

[7] Akimov A. Agafonova A. Analytical solutions for linear Volterra and Abel integral equations of second kind using a power series method, International Journal of Pure and Applied Mathematics, 105, No. 3, (2015), 529-535. doi:10.12732/ijpam.v105i3.18. 